\title{
Assessment of a child mental health inpatient unit
}

\author{
Objective measures of cost benefit should be the guide to effectiveness of function
}

\section{Introduction}

A child mental health inpatient service was never consistently available anywhere in Sri Lanka until February 2002, when a six-bed unit was opened at the Lady Ridgeway Hospital for Children (LRH) in Colombo. Though Sri Lankan health services are a long way from establishing a formal procedure for quality assessment and performance appraisal of clinical care, it is only right that we ask whether this new unit has served a useful purpose. Nearly 2 years after the inception of the inpatient unit, this article discusses our own investigations into its functioning.

\section{Low priority}

One may first ask why LRH, the premier tertiary care hospital for children and a leading academic centre for undergraduate and postgraduate training, failed to establish an inpatient mental health facility before 2002 . It is valid to ask such a question because there are advances in service development for child mental health care in India and many other parts of the world during the past several decades. Child mental health care originated with the child guidance concept in the middle of the last century. These child guidance clinics were entirely community based. Since then, there has been a gradual evolution into hospital based inpatient, outpatient and paediatric liaison services, which were facilitated mainly by two other parallel developments [1]. Firstly, improved knowledge has helped us to understand that many child mental health problems are neurodevelopmental and neuropsychiatric in nature. Secondly, and more importantly, there is an increasing public demand for better services [1]. Then, why did not Sri Lanka keep pace with developments in other parts of the world? The health authorities may argue that in a country that still battles with communicable diseases, with preventive services functioning below ideal levels of efficiency, priority is given to treatment of physical diseases and saving lives. Under the given circumstances, treatment of behavioural and emotional disorders, psychosocial risk management, promoting resilience and improving quality of life may come way down in their list of priorities. This is evident from the fact that the Department of Health allocates less than $1.5 \%$ of its health budget for mental health. Within that budget, there is no separate allocation for child mental health. This situation continues despite clear evidence that three out of 10 most burdensome diseases are mental disorders; that child disorders are of much higher prevalence in developing countries compared to developed countries; and that the World Health Organization emphasises that governments should take immediate steps to improve mental health services [2]. In Sri Lanka, the phrase "health needs" is often limited to a popular slogan among policy makers, and is not actually a guide to service development. Another likely reason is the negative attitude to mental illness and social stigma attached to it even when it concerns young children. It did not come as a surprise to us that an enclosing metal grill was offered for the current inpatient unit when it was originally planned. Though this idea was rejected outright, it shows the general prejudicial attitude; the belief that children admitted to the unit will run amok, and would need restraining to prevent them from harming other sick children.

\section{Who benefits from inpatient care?}

When a large majority can be effectively managed in an outpatient setting, what specific role can inpatient units play in the mental health care of children? Outpatient care alone may be sufficient when drug treatment is the mainstay in management and psychosocial care is not a primary need. In contrast, our own analysis of 191 consecutive admissions to the inpatient unit over a period of 15 months showed that $70 \%$ of parents and $26 \%$ of children needed psychological interventions as an essential part of their management. The children who received inpatient care belonged mainly to the broad diagnostic categories of emotional disorders $(53.9 \%)$, disruptive behavioural disorders $(25.1 \%)$ and developmental disorders (8.9\%). The main focus of psychological intervention was on improving competence of parents, facilitating conflict resolution and problem solving, and crisis intervention. Such strategies are difficult or impossible to offer in a busy outpatient setting where the time available is very limited. Available studies recognise the comprehensive and specific aspects of care that inpatient units can offer and their benefits [3-5]. 86.2\% of our patients too derived appreciable benefit from the interventions, as indicated by symptom reduction, improved functioning across several domains and positive feedback from parents. These improvements were sustained during the follow up period. 


\section{Was inpatient care cost effective?}

Inpatient care is costly, and in Sri Lanka a heavy financial burden is imposed on maintaining over-crowded wards in state hospitals. One way to make an inpatient unit cost effective is to achieve the desired health benefits in the shortest possible time. The average duration of stay in our inpatient unit was 6.4 days. This is shorter than the 2 to 6 week admissions reported from other similar units $[5,6]$. Limiting the period was possible because of the general policy of first screening all admissions, rapid decision making on management and active involvement of both medical and nursing staff in the assessment and therapy. This is in contrast to the widely recognised problem of indiscriminate admission of patients to tertiary care hospitals and slow decision making about disease management, which contribute to overcrowding and waste of resources [7]. However, our readmission rate was $12 \%$ and the outcome of management was judged unsatisfactory in $18.3 \%$ of patients. This may indicate that some discharges from the hospital were premature.

\section{Future directions}

Mental health problems affecting children are generally not as severe and disabling as those affecting adolescents. For instance, serious psychiatric illnesses such as schizophrenia and mood disorders first appear during adolescence but are rare in children. This may be used to support the argument that child mental health services can be exclusively outpatient based and that inpatient care is needed only for adolescents. However, the available evidence that child mental health problems and psychopathologies continue into adulthood [8] justify focused care at all possible levels. For example, there is a close connection between aggressive and disruptive behaviour in childhood with criminality and other antisocial tendencies in adulthood [9,10]; and untreated anxiety disorders in childhood will persist as disabling symptoms in adulthood [8]. Hence, the best opportunity for prevention lies in the evidence based interventions targeting children and parents. The large majority of children who received treatment in our unit had emotional and disruptive behavioural disorders with a potential for persistence of their problems if untreated. The other emerging trends for child mental health care in Sri Lanka are eating disorders and chronic physical disorders. Working in close collaboration with paediatricians in an inpatient setting is important in both. The liaison already established was evident from the fact that $53.4 \%$ of referrals to the inpatient unit were from paediatric medical and surgical units. Future developments should look at aspects that would contribute to improvement in knowledge and service development in the country. They include the training of medical and other health care personnel, database management, development of clinical practice guidelines and research.

\section{References}

1. Woolston JL. The administration of hospital based services. Child and Adolescent Psychiatry Clinics of North America 2002; 11: 43-65.

2. World Health Organization. Burden of mental and behaviour disorders. The World Health Report 2001. WHO, Geneva, 21-45.

3. Green J, Kroll L, Imrie D, Frances FM, Begum K, et al. Health gain and outcome predictors during inpatient and related day treatment in child and adolescent psychiatry. Journal of the American Academy of Child and Adolescent Psychiatry 2001; 40: 325-32.

4. Blanz B, Schmidt MH. Preconditions and outcome of inpatient treatment in child and adolescent psychiatry. Journal of Child Psychology and Psychiatry 2000; 41: 703-12.

5. Sourander A, Piha J. Three-year follow-up of child psychiatric inpatient treatment. European Child and Adolescent Psychiatry 1998; 7: 153-62.

6. Mayes SD, Calhoun SL, Krecko VF, Vvsell HP, Hu J. Outcome following child psychiatric hospitalization. Journal of Behavioral Health Services and Research 2001; 28: $96-103$.

7. Delpachithra C, Jayasinghe S. Overcrowding and "floor" patients in state hospitals: institutions can make a difference. Ceylon Medical Journal 2001; 46: 58-60.

8. Goodman R, Scott S. Epidemiology. In: Child Psychiatry. London: Blackwell Science, 1997: 30-38.

9. Stevenson J, Goodman R. Association between behaviour at 3 years and adult criminality. British Journal of Psychiatry 2001, 179: 197-202.

10. Satterfield JH, Schell A. A prospective study of hyperactive boys with conduct problem and normal boys: adolescent and adult criminality. Journal of the American Academy of Child and Adolescent Psychiatry 1997; 36: $1726-35$. 